\title{
LEARNING PROCESSES IN NETWORKED ENTERPRISES
}

Heinz-H. Erbe

Technische Universitaet Berlin

Institute of Vocational Education

Erbe0232@mailszrz.zrz.tu-berlin.de

The implementation of information and communication technology top-down do not guarantee for necessary organizational change. The paper discusses and introduces organizational learning processes in individual enterprises and networks in order to prepare for virtual enterprises and to establish a continuing improvement of work and learning processes to stabilize networks as the bases for such enterprises. The first steps, when using the developed learning loops with the management and the workforce of a small enterprise to prepare for networking, are presented.

\section{INTRODUCTION}

In the organization and management of enterprises, new models and theories have been developed that deviate significantly from earlier concepts. This applies to the structuring of activities as well as to the building of relations between individuals

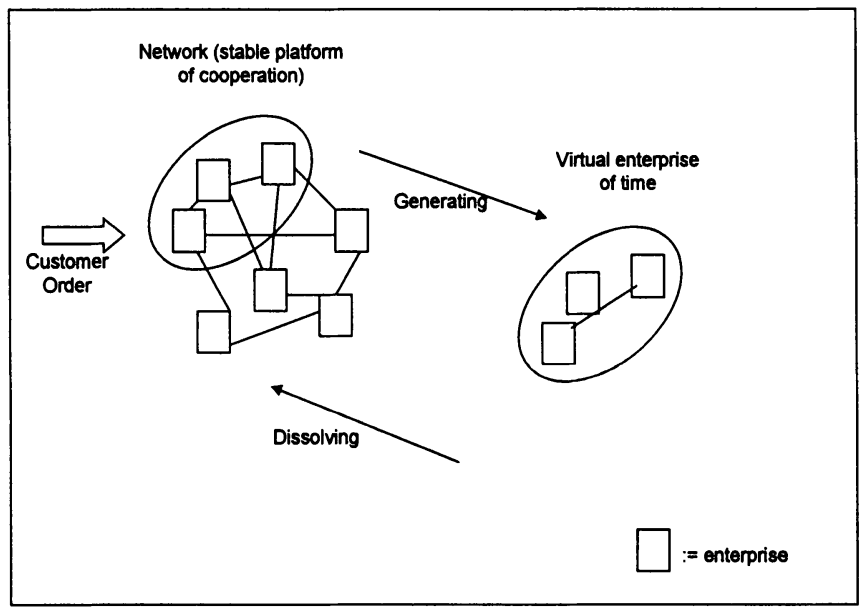

Figure 1 - Network and Virtual Enterprise (Schuh et.al, 1998) 
and organizations. One of these most promising models are Extended Enterprises or Virtual Enterprises (Schuh, Millar \& Goeranson, 1998; Zimmermann, 1997). Figure 1 illustrates the generation and dissolution of a virtual enterprise based on a stable platform, the networked enterprises. In order to assess and implement the new concepts into working methods and practices, new structures and working models must be created that involve new practices as well as new theories.

The virtual enterprise executes cooperatively the tasks belonging to the order of a customer, respecting the core competencies of the networked enterprises and using computer networks of information and communication technology. These technologies should be customized to the specific needs in networks. (CamarinhaMatos \& Afsarmanesh, 1999) discuss Cooperative Systems as a set of autonomous agents (computational and human) interacting which each other "through sharing their information, decision making capabilities and other resources, and distributing the corresponding workload among themselves, in order to achieve common or complementary goals". They emphasize the social and organizational issues and their consequences at technical level, the importance to identify the consequences in terms of the working structure, the processes, and the roles played by all social intervening actors. As small and medium sized enterprises (SMEs) are characterized "by a strong human-centered decision making philosophy, strong feelings of autonomy and information privacy" it is the challenge to overcome these cultural attributes to a new culture of willingness to build confidence and "a new idea of ownership that goes beyond the borders" of each participating enterprise within the network.

(Boekhoff \& Erbe, 1999) are stressing, that besides the implementation of technology, the success of an enterprise and networks as well, is determined mainly through the capability of management and workforce for cooperative work. It is an misunderstanding to assume that technology be an external force that would have deterministic impacts on organizational properties and structure.

\section{THE CHALLENGE OF LEARNING IN ENTERPRISES}

Small and medium sized enterprises are realizing that they will not survive if they do not change. To build networks, as a first step to virtual enterprises, using technologies to enhance vertical cooperation inside of an enterprise and horizontal, cross border, cooperation between enterprises is a challenge for individual and collective learning. Some aspects are to mention (Watkins \& Marsick, 1993):

- changes in technology that require learning new ways of thinking and cooperative working in order to build and to fully use computer-based skills.

- a service orientation that calls for learning to understand and satisfy customer needs.

- participatory management, which entails learning how to become appropriately involved in decision making, how to empower those with whom one works and how to ensure more responsibility for tasks that are less clear and less narrowly defined.

- high-performing and self-directing teams that necessitate learning how to work efficiently together in product development in close co-operation with the customer. 
- maintaining an almost full availability of equipment and manpower in order to fulfil scheduled delivery.

To foster these innovation processes and networked capabilities in SMEs a continuing formation of all members of the SMEs (managers, engineers and workers) is necessary. This is an understanding of a learning enterprise (Watkins \& Marsick, 1993; Pedler, Boydell \& Burgoyne, 1996). Many of the production oriented SMEs are unable to use all of their resources effectively, because the lack of collaboration among different professionals in the organization. These learning processes also generate other benefits like more cooperative and open work atmosphere, construction of most effective 'real' teams, a better quality system and a productive maintenance of manufacturing devices.

Together with an Innovation Center in Plauen/Vogtland, EastGermany, a concept got developed and carried out with all members of a small model making and rapid prototyping enterprise for operational further education of industrial staff including the management to prepare and accomplish technical innovation processes as well as networking with competitors and their effective realization in various models of labor organization. It was aimed to achieve the capability for integrating new technology and new forms of work organization. This concept was also aimed at enhancing the customer-supplier relation of the enterprise.

The concept suggests a learning helix (Erbe, 1998), in which the end of each loop differs qualitatively from the beginning of the helix. Each loop transits five steps (Figure 2). At the end of each loop the gained experiences and capacity to act is taken into comparison with the beginning of the loop to evaluate the learning success. Positive and negative results encourage further learning-loops. However, each loop needs to be entirely completed, as problems that occur in one loop shall not be transferred to the next. This would complicate or even block the following learning-loop.

\section{THE LEARNING LOOP}

Starting point for level 1 is the network of cooperative relations between the enterprises. An incoming order of a customer will be dealt with and processed through some enterprises belonging to the network respecting their core competencies. This leads to level 2 in Figure 2. Obtained experiences in working and learning when processing the customer order will be evaluated and discussed at levels (4) and (5) of the loop. Then beginning with (1) on a higher level the loop turns again. A coach or facilitator should support this process until it is established within the network.

\subsection{Level 1: from an individual enterprise to a network}

\section{(1) Definition of the present operational relations, Elaboration of aims}

Prior to making decisions on renewing once organization, the present state of an enterprises of the network shall be analyzed and evaluated.

Get in contact with each other, clarify relationships between one another, develop and accept attitudes and opinions, define objectives - through the definition of aims individuals become capable to act. 
Without visualizing and appreciating the actual assumptions of management, organization and learning, it is hardly possible to define methods which are effective, which shall be changed or improved. Hereby, the participation of the entire staff, in particular technicians and foremen is of major importance. Especially foremen are nowadays, in time of lean hierarchies, confronted with a high rate of uncertainty about their future tasks. However, to accomplish the transformation towards a new structure of tasks successfully, their operational experiences are required.

The management (CEO) is highly challenged, as they need to free themselves from the belief to control and even have to control the entire processes. They fear to render superfluous. On the contrary, they do not dare to overcome their own nature and delegate the competence of decision-making. Up to a certain company size a centralized organization structure may be effective. Above that, however, there is a critical moment in which the flexibility towards the customer and market requirements decreases. When this has been visualized and accepted by the entire staff, it is time to act.

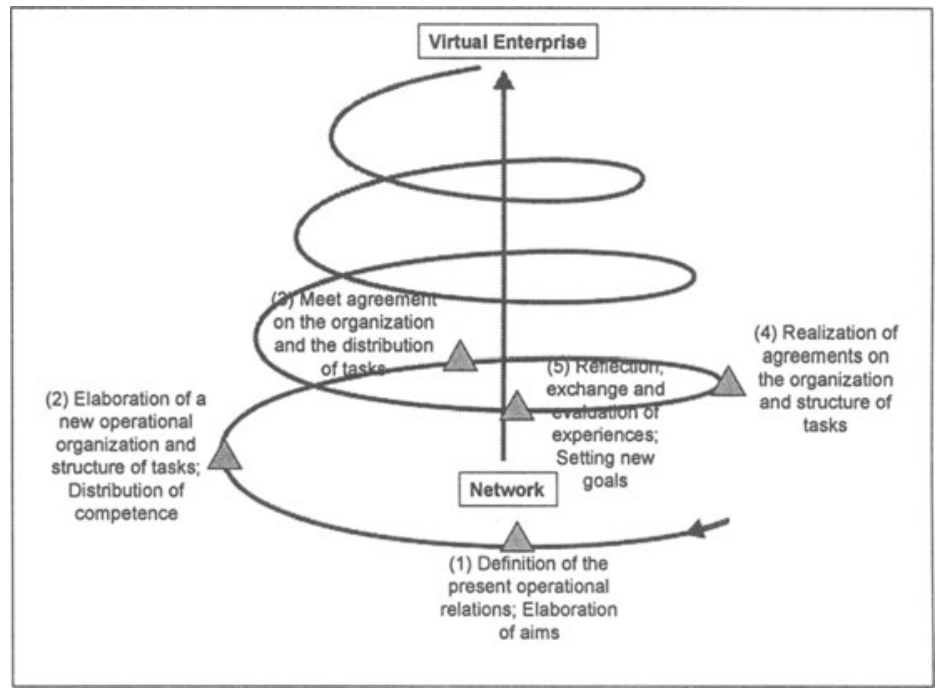

Figure 2 - Spiral Line of Learning

The delegation of the power of decision is paired with a learning process on both sides. This learning process needs to uncover and make aware of common opinions and beliefs. This process can be achieved with a mutual strategic planning and goal setting. A open, communicative and cooperative atmosphere, however, can only be realized, if the relationship between one another member of the organization is clearly defined.

With the development of strategic aims towards the customer or the market, most diverse beliefs can be cleared and freed in a open dialogue. Instead of a discussion, a dialogue can only be sufficient, if the members are conscious of their presumptions and are willing to overcome eventual unconscious individual defensive positions. 
Objective of learning / Result: Develop new attitudes and opinions and accept different attitudes and opinions; through the detailed definition of aims individuals become capable to act.

\section{(2) Elaboration of operational organization and structure of tasks,} Distribution of competence

After having elaborated joint aims in respect of necessary changes of the present operational organization with a new structure of tasks (in 1), the next step is to elaborate the distribution of competence and tasks within an enterprise and the network for all employees including the management.

The delimitation of competence and tasks shall rather support than disturb cooperative work. Nevertheless it is important to define limits, which can be dynamically rearranged.

Perfectionism, however, needs to be avoided as it acts as a creativity brake. Even the definition of responsibilities is problematic as it has a static effect and may secure the power of knowledge, which violates cooperative work severely.

In a dialogue with all members of the organization these arguments need to be reflected:

- Pre-assumptions need to be suspended;

- staff shall accept their members as colleagues

(there does not exist a status of superior / inferior staff members);

- a facilitator moderates the dialogue and stops it from being a discussion.

The facilitator is no member of the organization and is therefore not "blind". This is the basis for tasks of formation-, competence- and innovations centers.

Clarify tasks and competence with the staff to strengthen self-consciousness and accept the purpose of competence and task limits. Complete information about the total of operational processes and the structure of tasks is the main prerequisite.

Appreciating new roles (distribution of tasks) requires the acceptance of necessary change and of a broadened vision of the enterprise within the network. Therefore, potential knowledge about the aspired aims and strategies of the enterprise, its competitors and its market is obligatory. Daily information to the staff (shop floor information system, electrical planning board, e.g.) is as vital to meet the current goals sufficiently. Within daily meetings about task planning, this information should be discussed in the very detail. Information shall be available for everybody and shall not be filtered for a small defined task.

In terms of role-taking and the shift from the present role towards team-working, the period after the change of task structure needs to be taken into consideration by management (CEO) and staff.

Agreeing with the new requirements needs to be regarded by additional payments or further forms of gratification, it shall however not be regarded by superior roles. New forms of payment need to be arranged, whereby a mixture of individual recognition and team-benefit shall be developed. With constantly changing and possibly increasing operational demands for the staff there can easily be given an impression of exploitation or even self-exploitation, which is a tour of mountain ridges. Therefore, definite agreements on aims and the distribution of tasks may be helpful.

Criteria that validate the performance of staff need to be disclosed. Hereby, the management (CEO) is permanently challenged to a fair play with the staff. Within 
the new structure of tasks it shall be obvious to associate indirect labor with direct very closely. This necessitates extensive information about all operational processes and demands a transparency of decisions.

Objective of learning / Result: Acceptance of the distribution of competence and the structure of tasks in the totality of operational processes; definite and accepted distribution of tasks to minimize conflicts; internal and external handling of information about the enterprises and the network.

\subsection{Level 2: from network to virtual enterprises}

\section{(3) Agreements on the organization and the distribution of tasks within the virtual enterprise}

The new task structure was elaborated by all staff members and accepted (in 2). The bounds of competence are worked out; there is no one, who can and is supposed to do everything. Therefore exemptions have to be arranged since bounds change dynamically.

Furthermore these rules foster the conditions of cooperation. They are arranged and inter-link the present operational relations and the new structures of task. The arrangements consider the work flow, which has been worked out, the attainment and the vocational capacity of each staff member, their interest in personal development and by all means also theirs preferences and antipathy.

The arrangements about the rules of cooperation have to be documented, signed up by each member and finally published. Apparently these arrangement are valid until they are replaced by new arrangements. Gaining new experience may lead to modified arrangements in the next step of the loop. On the other hand, the clarification of the tasks within the development process will make these new arrangements inevitable.

Arrangements need to be put in concrete terms in order to rule out misunderstandings and interpretations. This is the only way to ascertain whether these arrangements were kept and whether they served the intended purpose. Variations within the organization are being realized and the arrangements will enhance the commitments.

Rules, how to deal with exceptions, e.g. the need to exceed competence boundaries, have to be arranged and therefore become apparent to all members. Arrangements structure the collective learning process within the enterprise or network. If the arrangements are written, they need to be taken down very concretely and precisely, for the possibility of misunderstandings and interpretations will increase, if the arrangements are being formulated too abstractly.

Objective of learning / Result: negotiate rules in order to structure the cooperation with regard to the idea of the dynamic boundaries (in 2); arrangements are put in terms, put down in writing, in order to find out further on whether they were kept; arrangement need to signed up by all members. Rules for exceptions have to be established.

(4) Realization of the agreements on the organization and structure of tasks of the virtual enterprise 
The lack of sufficient training combined with high expectations from the new structure of tasks may lead to frustration of the involved very quickly, especially since some staff members do not feel that they are able to meet the new demands. The firm has to invest into training, which also shows that the enterprise is interested in the individual development of the employees. Professional training combined with team-learning is required to develop decisive competence within the new structure of tasks. It is a mutual learning process - decision-makers learn to delegate competencies and furthermore to waive capacious control of the execution, otherwise the flexible structure, which is necessary, will never develop. All members need to learn to cope with mistakes.

Instead of a further vocational education out of the shopfloor, a permanent further education within the workshop-level is needed. New career understanding and perspective need to arise with new incentives, which prepare for these pretentious tasks. As yet, the traditional further education gives the incentive to get out of the workshop. But these jobs will not exist in future anymore.

No one can be forced to inter-link his personal development, personal mastery with the development of the operative work flow. Personal development and mastery has to arise by itself - the coupling with the enterprise interest depends heavily on the organization of work.

Compelling staff members with training, which imposes the view of the management, will lead to nothing. The challenge (task) is to unleash both knowledge and skills of the members. Personal Mastery can only arise and develop to the full, if combined with the empowerment for own decisions.

The learning process consists in becoming aware of the risks both the management and the staff. Both sides need the possibility to overcome the prejudices and defense routines, and to assume responsibility, which changes the task design and supports to realize the joint aims and enhances the personal mastery. It is of great importance that decisions are worked out together and therefore the responsibility is taken by the entire group. Teamwork, in which the group leader decides all by himself and the group has to assume the responsibility of this decision, is therefore no teamwork.

Objective of learning / Result: Take over of personal, delimited and arranged responsibility and decision sphere; realization and surmounting of internal and external resistance; gain of self-confidence and confidence in agreed aims.

\section{(5) Reflection, exchange and evaluation of experiences; Setting new aims for the network}

In order to pass one loop, an analysis of the experience of the last four stations is necessary. This evaluation has to be carried out by all staff members in order to realize success and to name failures. It is unlikely, that all joint aims like organizational change, new structure of tasks, delegation of competencies can be reached by passing the loop for the first time without any problems. It may happen, that these joint aims will never be reached. But nonetheless all personnel involved will realize, that the change was initiated by themselves, by their way-of-thinking, by their action. Individual experience is being exchanged and especially members become aware of collective experience. People involved become aware that stagnation or development is a result of their action and therefore of their personal 
responsibility - the necessity of arrangements and the question how to deal with them. Without commitment and agreement, both allowing to verify, there will not be a change in the structure of decisions.

The evaluation of the experience serves as a preparation for the next loop, not only in terms of a permanent improving process but also in order to reach new aims, which is a consequence of new tasks.

Objective of learning / Result: Recognition and evaluation of success and failure; elaboration of the necessity and expediency of agreements; correction of the innovation process; orientation towards new aims for the network.

\section{CONCLUSION}

The learning process proceeds in loops and passing one loop completely describes one complete action. Based on the experiences of the former loops, a learning process starts, which leads to a permanent development of not only a personal but also a collective mastery. Metaphorically speaking, the learning loops will move up like a helix. This process is only carried out by the individuals involved. But after implementing such a process, individuals may leave, but the process will not collapse. This is the aim of the learning process, that combines the individual with the collective (Senge, 1990).

As was expected, apart from the Innovation Center, also the participating university got a better understanding of requirements of SMEs, because in its teaching contents and teaching structure it is often far away from economic and managerial needs in SMEs. So far it was a goal to develop new forms of cooperation in continuing formation considering that new technologies as information and communication technologies got transferred to SME's, but to use them effectively has to be elaborated and steadily improved with all the personnel of the enterprise, management and workforce.

\section{REFERENCES}

1. Boekhoff, H. and Erbe, H.-H. Organisationales Lernen: kritischer Erfolgsfaktor fur virtuelle Unternehmen? In: Industrie Management 15, Heft 6, 1999, pp. 13 - 16.

2. Camarinha-Matos, L.M. and Afsarmanesh, H. Cooperative Systems Challenges in Virtual Enterprises. In: Proceedings Esprit project 22647, Prodnet II, 1999.

3. Erbe, H.H. Innovationsprozesse in Betrieben - ein Arbeitsfeld betrieblicher Bildung. In: Die Modernitaet des Unmodernen. (Schuette, F. and E. Uhe eds.), Bundesinstitut fuer Berufsbildung, Berlin, 1999, pp. 395 - 406.

4. Pedler, M., Boydell, T. and J. Burgoyne. The Learning Company. MacGraw-Hill, London, 1996.

5. Schuh, G., Millarg, K. and Goransson, A. Virtuelle Fabrik - Neue Marktchancen durch dynamische Netzwerke. C. Hanser Verlag, München, 1998.

6. Senge, P. The Fifth Discipline: the art and practise of the learning organization. Doubleday, NewYork, 1990.

7. Watkins, Karen E. and Marsick, Victoria J. Sculpting the Learning Organization. Jossey-Bass Publishers, San Francisco, 1993.

8. Zmmermann, F.O. Structural and Managerial Aspects of Virtual Enterprises. University of Siegen, Germany, 1997, http://www.uni-siegen.de/others/student/vwi/vision/it1.htm 